\title{
Minimizing Radiation Exposure in Evaluation of Pediatric Head Trauma: Use of Rapid MR Imaging
}

\author{
H. Mehta, J. Acharya, A.L. Mohan, M.E. Tobias, L. LeCompte, and D. Jeevan
}

\begin{abstract}
BACKGROUND AND PURPOSE: With $>473,000$ annual emergency department visits for children with traumatic brain injuries in the United States, the risk of ionizing radiation exposure during CT examinations is a real concern. The purpose of this study was to assess the validity of rapid MR imaging to replace CT in the follow-up imaging of patients with head trauma.
\end{abstract}

MATERIALS AND METHODS: A retrospective review of 103 pediatric patients who underwent initial head CT and subsequent follow-up rapid MR imaging between January 2010 and July 2013 was performed. Patients had minor head injuries (Glasgow Coma Scale, >13) that required imaging. Initial head CT was performed, with follow-up rapid MR imaging completed within 48 hours. A board-certified neuroradiologist, blinded to patient information and scan parameters, then independently interpreted the randomized cases.

RESULTS: There was almost perfect agreement in the ability to detect extra-axial hemorrhage on rapid MR imaging and CT $(\kappa=0.84$, $P<$.001). Evaluation of hemorrhagic contusion/intraparenchymal hemorrhage demonstrated a moderate level of agreement between $M R$ imaging and $\mathrm{CT}(\kappa=0.61, P<.001)$. The ability of MR imaging to detect a skull fracture also showed a substantial level of agreement with $\mathrm{CT}(\kappa=0.71, P<.001)$. Detection of diffuse axonal injury demonstrated a slight level of agreement between MR imaging and CT $(\kappa=0.154$, $P=$.04). However, the overall predictive agreement for the detection of an axonal injury was $91 \%$.

CONCLUSIONS: Rapid MR imaging is a valid technique for detecting traumatic cranial injuries and an adequate examination for follow-up imaging in lieu of repeat CT.

ABBREVIATION: $r M R I=$ rapid MR imaging

$\mathrm{H}$ ead trauma continues to be a leading cause of death and disability in children in the United States. ${ }^{1}$ Every year, $>473,000$ visits to the emergency department are related to brain injury, ${ }^{2}$ most resulting from minor injuries or falls. Although most head injuries are classified as mild, approximately $10 \%-15 \%$ of children sustain a severe one. The incidence of intracranial injury following minor head trauma is unknown; however, with increasing public awareness of traumatic brain injury and concussion, there has been a rise in research of minor head injuries. Methods of diagnosis, ${ }^{3,4}$ hospital admission criteria, ${ }^{5,6}$ and return-to-play criteria ${ }^{7,8}$ are a few of the active areas of research.

Children with head trauma, at risk for intracranial injury, should be initially imaged with $\mathrm{CT}^{9}$ because it remains the crite-

Received March 31, 2015; accepted after revision May 20.

From the Departments of Radiology (H.M., J.A., L.L.) and Neurosurgery (A.L.M., M.E.T., D.J.), New York Medical College, Valhalla, New York.

Please address correspondence to Hasit Mehta, MD, New York Medical College, Department of Radiology, 100 Woods Rd, Valhalla, NY 10595; e-mail:

MehtaH@wcmc.com

http://dx.doi.org/10.3174/ajnr.A4464 rion standard technique for the evaluation of head trauma. ${ }^{10} \mathrm{Al}-$ though the incidence of injuries requiring neurosurgical intervention in children with minor head injuries is low, the use of CT for evaluation has been increasing. The use of CT increased from $13 \%$ to $22 \%$ from 1995 to 2003 , with a peak of $29 \%$ in $2000 .{ }^{11}$ The decision to obtain neuroimaging for children with minor head trauma must balance the importance of identifying head injuries with the risks of CT. There is growing awareness in the medical community and public of increased cancer risk caused by ionizing radiation. ${ }^{12}$ Brenner et al $^{13}$ estimated that 170 additional fatal cancers will develop due to head CT examinations performed in children younger than 15 years of age in the United States in a single year. In addition, some children may require sedation to obtain an adequate CT examination, which can be associated with as high as a $20.1 \%$ chance of an adverse event. ${ }^{14}$

MR imaging is an alternative technique that avoids ionizing radiation exposure altogether and produces high-quality images. A study with conventional sequences requires long acquisition times and is susceptible to motion artifacts. The need for sedation increases the risk to the patient, lengthens the time needed to 
acquire patient images, and further increases the cost of standard MR imaging. ${ }^{14,15}$

Modified MR imaging protocols with reduced acquisition times have been used successfully in non-neurosurgical patients, ${ }^{16,17}$ and rapid MR imaging (rMRI) or "quick-brain" MR imaging protocols have become an accepted technique to evaluate and follow patients with hydrocephalus. ${ }^{18-20}$ Missios et $\mathrm{al}^{21}$ investigated the use of rMRI in patients without hydrocephalus and concluded that it was an adequate neuroimaging tool for evaluation and follow-up. The use of rMRI protocols in evaluating pediatric patients with minor head injuries remains to be validated.

As far as we are aware, a systematic search of current literature did not yield a previous study examining the validity of rMRI in the imaging of pediatric patients with head trauma. The purpose of our study was to demonstrate the efficacy of replacing ionizing CT imaging with nonionizing rMRI for follow-up of patients with minor head trauma.

\section{MATERIALS AND METHODS}

Our institutional review board approved this study, with a waiver of informed consent. All CT and MR imaging examinations were performed as a standard of care; the results were retrospectively reviewed. The study protocol complied with the Health Insurance Portability and Accountability Act.

\section{Patient Group}

Patients evaluated at Westchester Medical Center, a level 1 pediatric trauma center, between January 2010 and July 3013 were screened for eligibility; inclusion criteria were patients presenting with minor head injury (Glasgow Coma Scale, $>13$ ) who were evaluated with rMRI performed within 48 hours following an initial CT. Patients were selected for CT on the basis of regional emergency department criteria for imaging, and rMRI was completed at our institution regardless of CT findings.

\section{Imaging Protocol}

CT studies performed at our institution used a Brilliance 64detector row CT scanner (Philips Healthcare, Best, the Netherlands); images were acquired helically and reconstructed into a contiguous 3-mm axial dataset acquired from the base of the skull to the vertex. Images obtained at an outside institution were uploaded to our system as per our protocol for interpretation of outside imaging studies. Images obtained from outside institutions used their own protocol for image acquisition; minimum imaging requirements for outside studies included datasets acquired from the base of the skull to the vertex with contiguous axial images of $\leq 5 \mathrm{~mm}$.

rMRI examinations were performed by using 1.5T (Achieva 1.5T; Philips Healthcare) and 3T (Achieva 3T X; Philips Healthcare) scanners. rMRI sequences included the following: axial single-shot T2 fast-field echo EPI: 5-second scanning time; TR, 2000 ms; TE, 25 ms; axial single-shot diffusion-weighted imaging: 35second scanning time; TR, $3000 \mathrm{~ms}$; TE, $65 \mathrm{~ms}$; axial single-shot FLAIR: 45-second scanning time; TR, 12,000 ms; TI, $2850 \mathrm{~ms}$; TE, 135 ms; axial T2 fast-field echo: 35-second scanning time; TR, 550 ms; TE, 15 ms; coronal T2 turbo spin-echo: 35-second scanning time; TR, $3500 \mathrm{~ms}$; TE, $80 \mathrm{~ms}$. Axial T2 turbo spin-echo (30- second scanning time; TR, $3000 \mathrm{~ms}$; TE, $80 \mathrm{~ms}$ ) may be performed if desired by the radiologist or MR imaging technologist, with a total scanning time of 2.5-3 minutes (Fig 1: rMRI sequences obtained).

\section{Image Interpretation}

CT and rMRI studies that met the inclusion criteria were randomized, then independently and retrospectively evaluated by a board-certified radiologist, with added board certification in neuroradiology, on a digital PACS workstation. The studies were retrospectively reviewed during a 3-month period from October 2013 to January 2014. The reader was blinded to patient-identifying information and imaging parameters. Furthermore, all CT and rMRI studies were reviewed independent of one another.

During interpretation, the radiologist evaluated the presence or absence of the following findings: extra-axial hemorrhage (subdural, epidural, or subarachnoid hemorrhage), hemorrhagic contusion/intraparenchymal hemorrhage, calvarial fracture, and/or diffuse axonal injury. The radiologist was also asked to comment on the presence of motion degradation.

\section{Statistical Evaluation}

We performed statistical evaluation of the data by using commercially available statistical software (SPSS; IBM, Armonk, New York). Cohen $\kappa$ statistics ${ }^{22}$ were performed to determine whether there was agreement between the 2 imaging modalities as to the presence or absence of extra-axial hemorrhage, hemorrhagic contusion/intraparenchymal hemorrhage, fracture, and/or diffuse axonal injury. Positive and negative percentage agreement and overall percentage agreement of the findings on CT and rMRI were also analyzed.

\section{RESULTS}

A total of 103 pediatric patients presenting with minor head trauma (presenting Glasgow Coma Scale, $>13$ ) underwent rMRI following initial CT as per regional head trauma injury criteria. Two patients were excluded because the rMRI was performed following surgical intervention for intracranial hemorrhage. The mean age of the 101 subjects was 6 years (range, $0-19$ years); there were 57 males (55\%) and 46 females (45\%). The most common mechanism of injury was a fall. rMRI was performed within 48 hours of arrival at our institution and CT imaging; the average time between initial CT and follow-up rMRI was 19 hours. No patient received anesthesia for the study.

On review of the imaging, 24 patients had some degree of motion degradation on rMRI compared with only 7 patients on CT. Only 4 patients had evidence of motion degradation on both CT and rMRI. The degree of motion artifacts on the examinations was mild to moderate and not enough to require exclusion from the study because the studies were deemed diagnostic. Overall, the correlation of traumatic findings between initial CT and rMRI was $\kappa=0.73(P<.001 ; 95 \% \mathrm{CI}, 0.88-0.94)$. This indicates a substantial agreement in findings between imaging modalities (Table 1). An overall percentage agreement of $92 \%$, positive percentage accuracy of $91 \%$, and negative predictive accuracy of $94 \%$ further demonstrated this agreement.

When one looks at subtypes of injuries, the ability to detect 


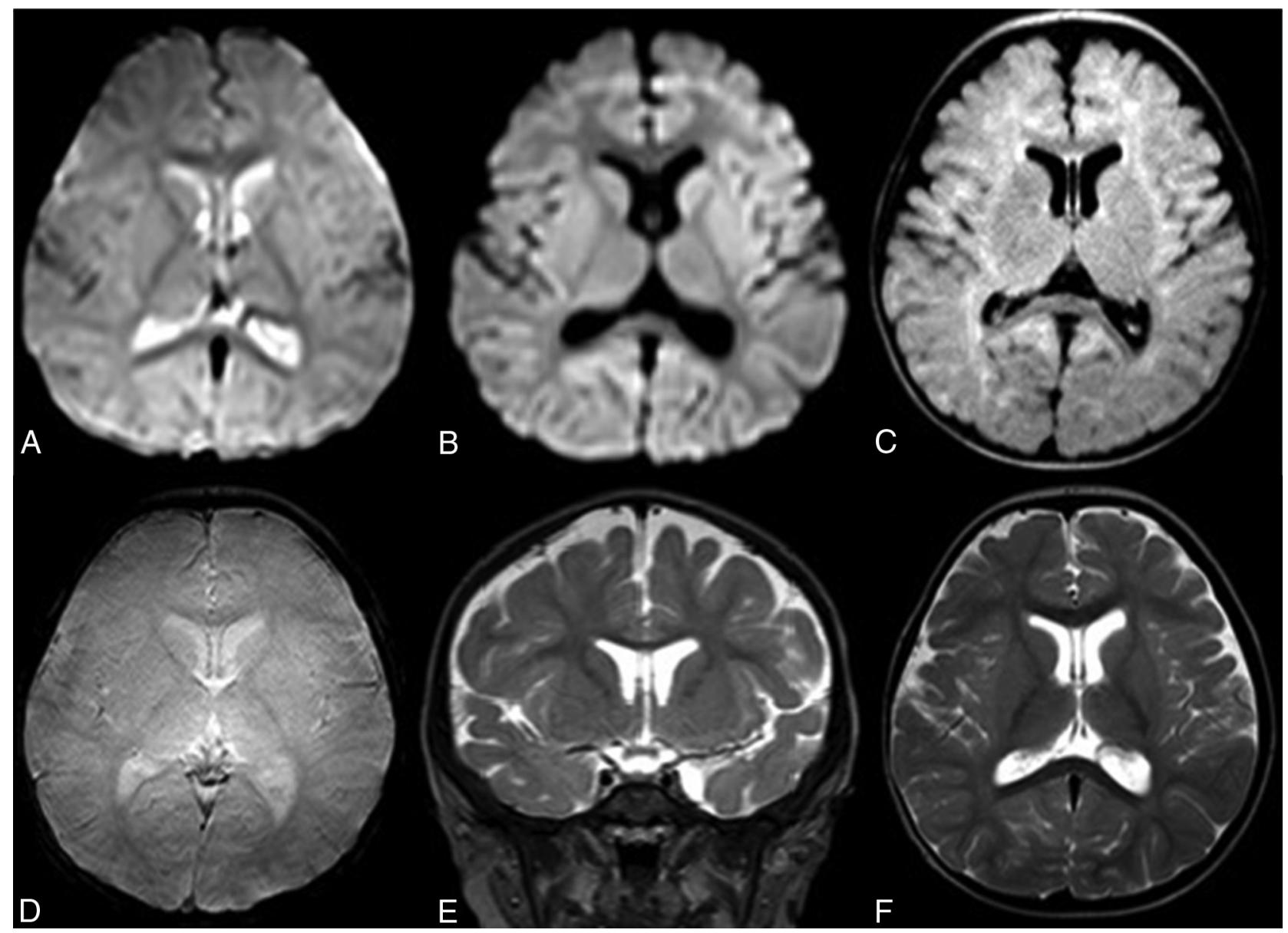

FIG 1. Sample images from a routine rMRI examination. Axial single-shot T2 fast-field echo echo-planar (A), axial single-shot diffusion-weighted $(B)$, axial single-shot FLAIR (C), axial T2 fast-field echo (T2*) (D), coronal T2 TSE (E), and axial T2 TSE (F) images.

Table 1: Presence of a positive image finding following minor head injury ${ }^{\mathrm{a}}$

\begin{tabular}{lccc}
\hline \multirow{2}{*}{$\begin{array}{c}\text { Positive } \\
\text { Scan }\end{array}$} & \multicolumn{2}{c}{ CT } & \\
\cline { 2 - 3 } Findings & Negative & Positive & Total \\
\hline rMRI & & & \\
Negative & 22 & 10 & 32 \\
Positive & 1 & 68 & 69 \\
Total & 23 & 78 & 101 \\
\hline
\end{tabular}

${ }^{a} \kappa$ measure of agreement, $0.728 ; P<.001$; standard error, 0.075 ; overall percentage agreement, $92 \%$; positive percentage agreement, $91 \%$; negative percentage agreement, $94 \%$.

extra-axial hemorrhage (epidural, subdural, subarachnoid hemorrhage) on CT and rMRI was comparable, with a $\kappa=0.84$ $(P<.001 ; 95 \% \mathrm{CI}, 0.74-0.95)$. With an overall percentage agreement of $92 \%$ and a positive percentage agreement of $91 \%$ and a negative percentage agreement of $94 \%$, the ability to detect extraaxial hemorrhage on rMRI was almost in perfect agreement with findings on CT (Table 2).

Although there was substantial agreement between rMRI and CT on the presence of hemorrhagic contusion/intraparenchymal hemorrhage (Table 3), the correlation was not as great as that for the presence of extra-axial hemorrhage, with $\kappa=0.61(P<.001$; 95\% CI, 0.42-0.80). However, when we looked at the results, there was a high positive predictive agreement of findings on rMRI (93\%), with a high overall percentage agreement $(87 \%)$.
Table 2: Presence of extra-axial hemorrhage following minor head injury ${ }^{\mathrm{a}}$

\begin{tabular}{lccc}
\hline \multirow{2}{*}{$\begin{array}{c}\text { Extra-Axial } \\
\text { Hemorrhage }\end{array}$} & \multicolumn{2}{c}{ CT } & \\
\cline { 2 - 3 } & Negative & Positive & Total \\
\hline rMRI & & & \\
Negative & 44 & 5 & 49 \\
Positive & 3 & 49 & 52 \\
Total & 47 & 54 & 101 \\
\hline
\end{tabular}

${ }^{a} \kappa$ measure of agreement, $0.841 ; P<0.001$; standard error, 0.054 ; overall percentage agreement, $92 \%$; positive percentage agreement, $91 \%$; negative percentage agreement, $94 \%$.

Table 3: Presence of a contusion/intraparenchymal hemorrhage following minor head injury ${ }^{\mathrm{a}}$

\begin{tabular}{lccc} 
& \multicolumn{2}{c}{ CT } & \\
\cline { 2 - 3 } Contusion & Negative & Positive & Total \\
\hline rMRI & & & \\
Negative & 74 & 1 & 75 \\
Positive & 12 & 14 & 26 \\
Total & 86 & 15 & 101 \\
\hline
\end{tabular}

${ }^{a} \kappa$ measure of agreement, 0.609; $P<.001$; standard error, 0.095; overall percentage agreement, $87 \%$; positive percentage agreement, 93\%; negative percentage agreement, $86 \%$.

The negative percentage agreement was also high at $86 \%$. It appears that rMRI detects more contusive changes than initially seen on CT and may be more sensitive in detecting intraparenchymal blood. 
A similar finding was noted when looking at the presence of diffuse axonal injury (Table 4). There was only slight agreement for the presence of axonal injury between rMRI and CT with $\kappa=$ 0.15 ( $P=.04$; 95\% CI, $-0.15-0.45)$. However, the overall percentage agreement was $91 \%$, and the negative percentage agreement was $92 \%$. The positive percentage agreement was only $50 \%$, with 8 cases in which rMRI-suspected axonal injury was not seen on CT. The relatively low positive percentage agreement may be secondary to increased sensitivity to this injury type with the use of rMRI, especially because only a single case had a description of positive axonal injury seen on CT that was not seen on rMRI.

Even though it was predicted that CT would be more reliable in detecting skull fractures compared with rMRI, this reliability was not demonstrated (Table 5). The reliability of rMRI to detect skull fractures was found to be $\kappa=0.71(P<.001 ; 95 \% \mathrm{CI}$,

Table 4: Presence of diffuse axonal injury following minor head injury $^{\mathbf{a}}$

\begin{tabular}{lccr}
\hline $\begin{array}{l}\text { Diffuse } \\
\text { Axonal } \\
\text { Injury }\end{array}$ & \multicolumn{2}{c}{ CT } & \\
\cline { 2 - 3 } & Negative & Positive & Total \\
\hline rMRI & & & \\
Negative & 91 & 1 & 92 \\
Positive & 8 & 1 & 9 \\
Total & 99 & 2 & 101 \\
\hline
\end{tabular}

${ }^{a} \kappa$ measure of agreement, $0.154 ; P=.039$; standard error, 0.153 ; overall percentage agreement, $91 \%$; positive percentage agreement, $50 \%$; negative percentage agreement, $92 \%$.

Table 5: Presence of a skull fracture following minor head injury ${ }^{\mathrm{a}}$

\begin{tabular}{lccc}
\hline \multirow{2}{*}{$\begin{array}{c}\text { Skull } \\
\text { Fracture }\end{array}$} & \multicolumn{2}{c}{ CT } & \\
\cline { 2 - 3 } & Negative & Positive & Total \\
\hline rMRI & & 12 & \\
Negative & 44 & 42 & 56 \\
Positive & 3 & 54 & 45 \\
Total & 47 & 101 \\
\hline
\end{tabular}

${ }^{a}{ }^{\mathrm{a}}$ measure of agreement, $0.705 ; P<.001$; standard error, 0.069 ; overall percentage agreement, $85 \%$; positive percentage agreement, $78 \%$; negative percentage agreement, $94 \%$.
0.56-0.84), indicating substantial agreement. The overall percentage agreement was $85 \%$, with a negative percentage agreement of $94 \%$ and a positive percentage agreement of $78 \%$. In 12 cases, rMRI failed to detect a skull fracture seen on CT. None of these cases necessitated neurosurgical intervention.

\section{DISCUSSION}

The results of this study demonstrate that rMRI can detect traumatic injuries with a similar sensitivity and specificity compared with CT in the setting of minor head injuries. This finding was particularly true in the detection of extra-axial hemorrhage and intraparenchymal contusion (Fig 2; extra-axial hemorrhage). In the case of intraparenchymal hemorrhage, only a single patient had a positive finding that was not detected on rMRI. Conversely, however, 12 patients had contusive changes identified on rMRI, which were not seen on CT. This finding may simply be a reflection of the ability of the rMRI sequences to detect the presence of blood with increased sensitivity (Fig 3; left temporal lobe contusion not seen on CT but visualized on follow-up rMRI). We found that the T2 fast-field echo EPI sequence was most useful in appreciating acute blood products due to the susceptibility effects from deoxyhemoglobin. The coronal T2 TSE was also particularly useful for detecting smaller convexity blood products and traumatic brain injury at the inferior frontal lobes.

While it had been previously thought that rMRI would be insensitive for detecting skull fracture, ${ }^{23}$ our study did not reflect that supposition. Of the 101 patients, only 12 had a skull fracture found on CT that was not identified on rMRI. However, as with other clinical studies, none of these skull fractures required surgical intervention (Fig 4; fracture, CT and rMRI). ${ }^{24}$ The fractures not identified on rMRI typically were nondepressed skull fractures. Although these fractures were not identified on rMRI when the reader was blinded to the initial CT, when they were retrospectively reviewed with the initial head $\mathrm{CT}$, most fractures were identified as in the case shown in Fig $4 F,-G$.

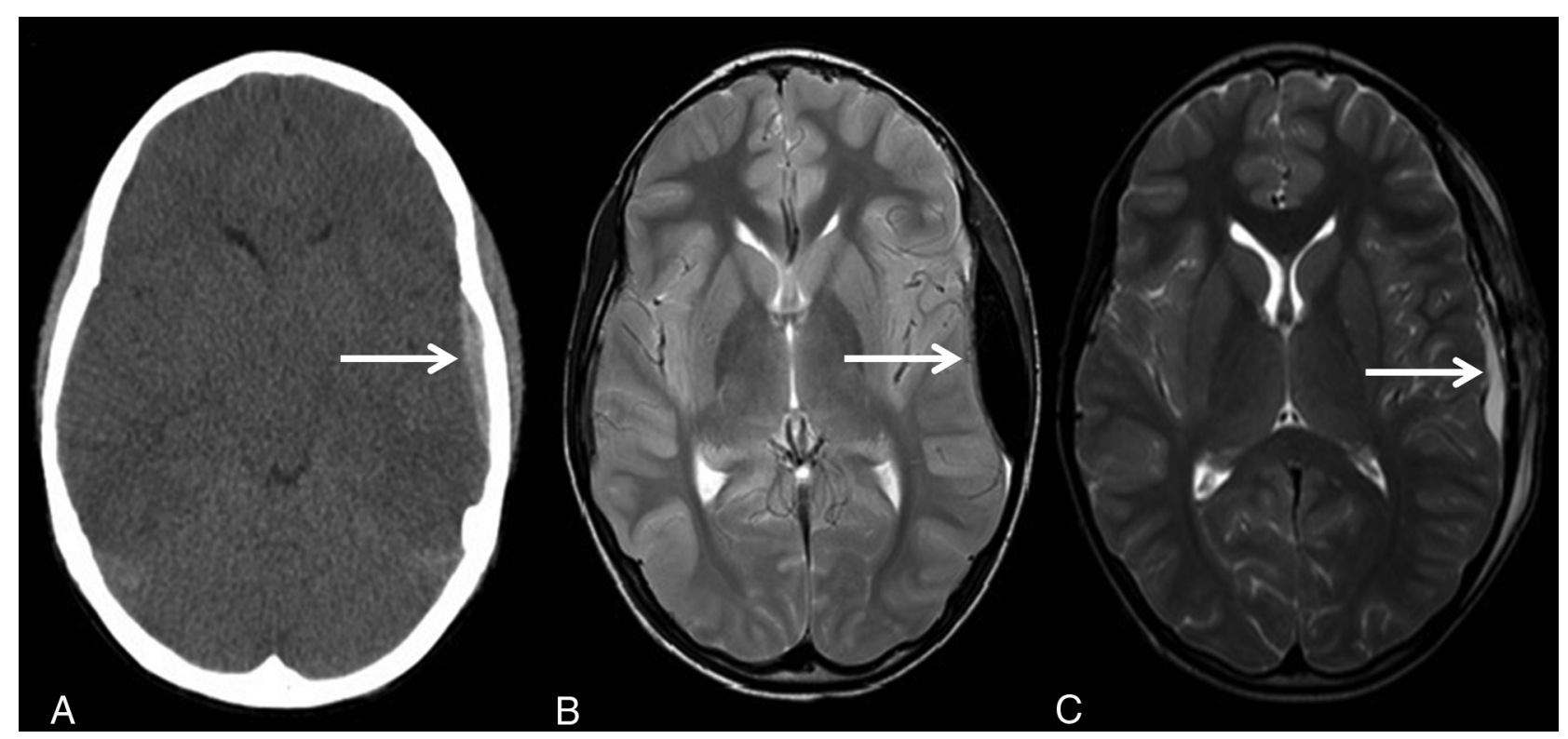

FIG 2. An 8-year-old child who fell from a bike. Initial noncontrast head CT shows a left epidural hemorrhage ( $A$ ). Follow-up rMRI axial T2 TSE shows interval-increased size of the left epidural hemorrhage (B). Follow-up rMRI axial T2 TSE shows interval craniotomy and evacuation of the left epidural hemorrhage (C). 


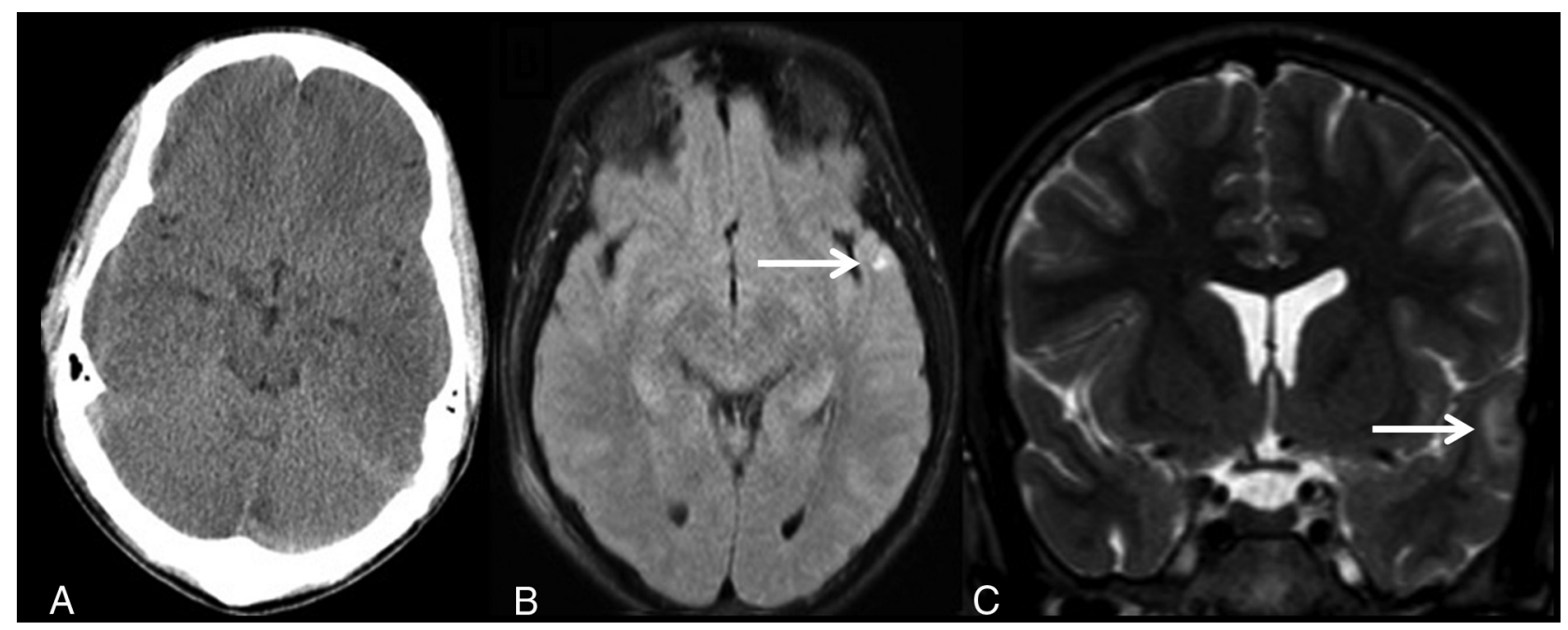

FIG 3. A 16 -year-old pedestrian struck by an automobile. Negative noncontrast CT of the head (A), axial single-shot FLAIR (B), and coronal T2 TSE (C) demonstrate a small left temporal lobe contusion.

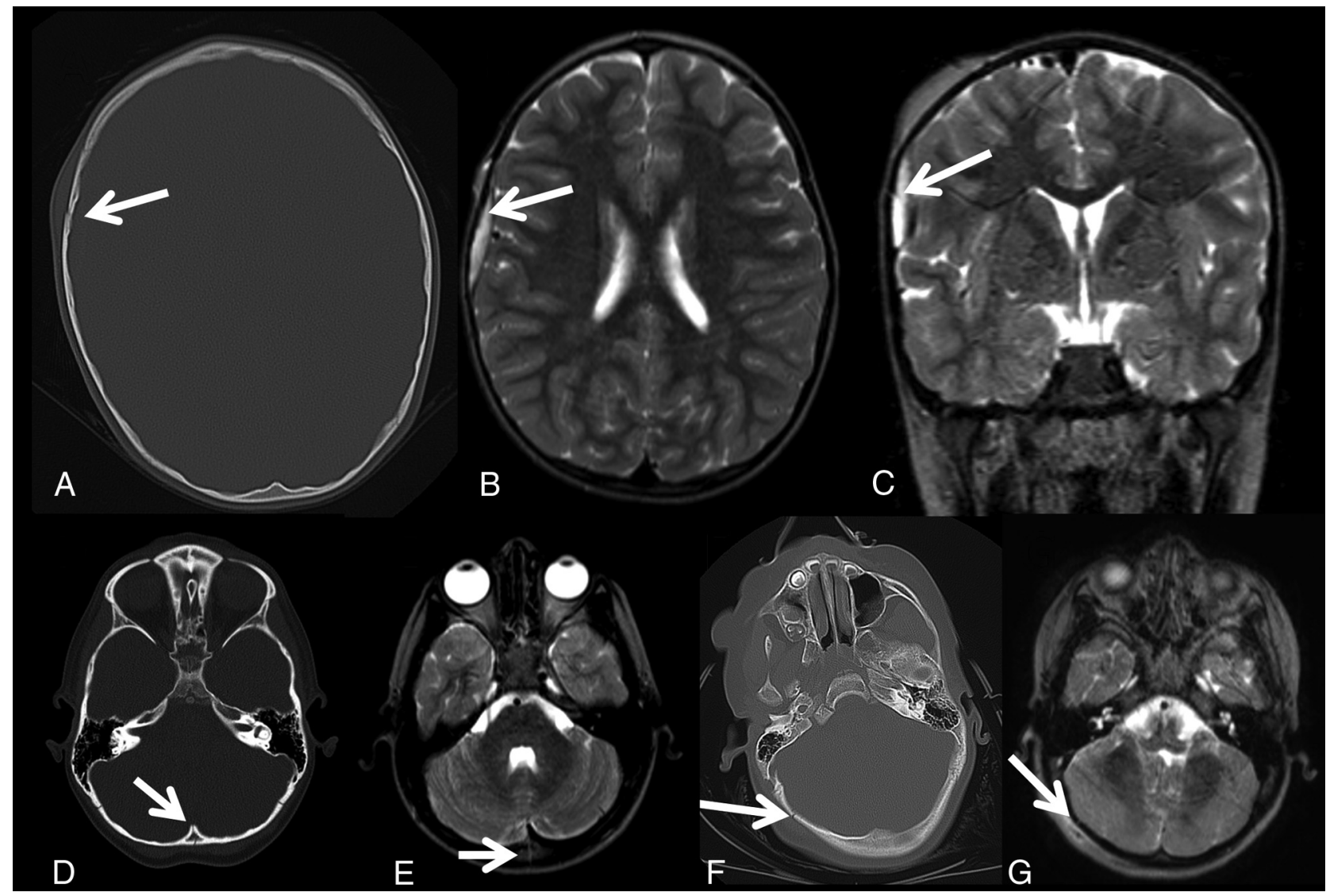

FIG 4. Axial noncontrast head $C T(A)$ demonstrates a right frontal bone fracture. On the same patient, a right frontal extra-axial hemorrhage with fracture is present on the axial T2 TSE (B) and coronal T2 TSE (C). Axial noncontrast head CT (D) shows an occipital fracture with a corresponding fracture seen on the axial T2 TSE sequence $(E)$. Axial noncontrast head CT $(F)$ shows the right occipital fracture. The fracture was not identified prospectively on rMRl; however, it can be identified retrospectively on $\mathrm{rMRI}(G)$ when read in conjunction with the initial head $C T$.

In the detection of diffuse axonal injury/shear injuries, there was a very clear difference between rMRI and CT. Although a significant correlation and a high negative percentage agreement were found, there was a comparatively low positive percentage agreement, with 8 cases having a positive rMRI interpretation compared with CT. This finding as in the case of detection of contusive injuries, may simply reflect the higher sensitivity of MR imaging to these injuries, as previously demonstrated in other studies. ${ }^{25-29}$ The time delay between initial CT and follow-up rMRI could also result in increased conspicuity of axonal injury due to interval blossoming. Additionally, DWI and FLAIR imaging may be more sensitive for the detection of diffuse axonal in- 


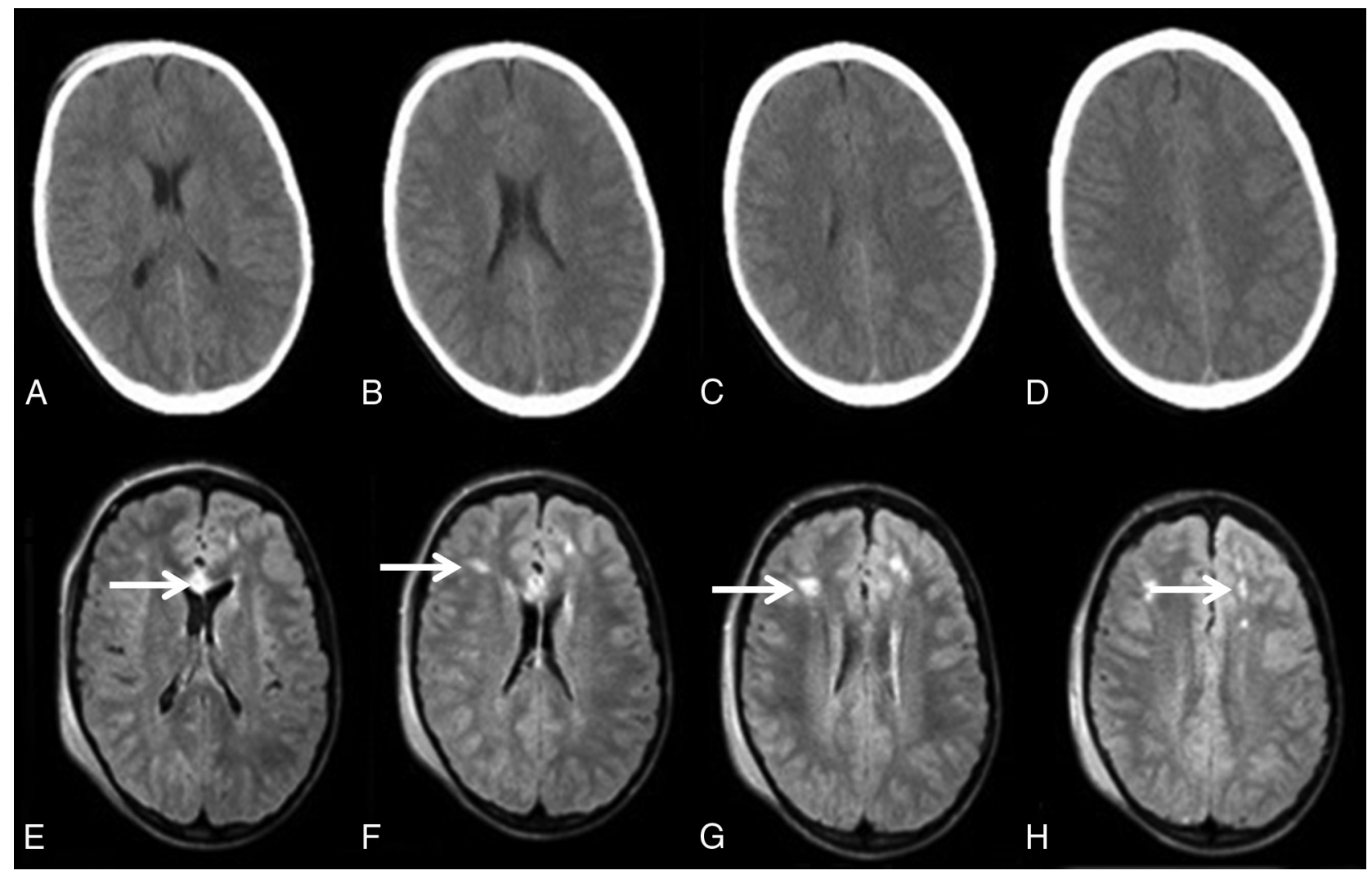

FIG 5. A 15 -year-old adolescent involved in an all-terrain vehicle rollover. Axial noncontrast head CT images (A-D) demonstrate no abnormal finding. The axial single-shot FLAIR images $(E-H)$ demonstrate multiple foci of abnormal signal in the frontal white matter and genu of the corpus callosum, compatible with diffuse axonal injury.

jury and parenchymal contusion compared with CT. An example of the increased sensitivity of FLAIR imaging to detect diffuse axonal injury is demonstrated in the case example shown in Fig 5, in which a pediatric patient with a minor head injury demonstrated multiple FLAIR signal changes not detected on CT (Fig 5; axonal injury, CT and rMRI).

Most reports have focused on the use of rMRI in the evaluation of hydrocephalus; however, other disorders may also be successfully imaged with this technology. CT has increasingly become part of the routine algorithm in the setting of trauma, particularly given its speed of acquisition. In this population, rMRI has been primarily used for follow-up imaging, ${ }^{21}$ even though the sensitivity and specificity of rMRI for various findings that influence the medical and surgical management of cranial trauma have yet to be firmly established. Previous studies that have looked at the use of MR imaging in patients with trauma have focused on prognostication $^{30}$ and, as such, have included full-sequence studies, often completed several weeks after the injury. A strength of our study is the short duration between the 2 modalities, with an average time between the acquisition of CT and rMRI of 19 hours, with the longest duration $<48$ hours.

Only one other study has attempted to validate the use of rapid-sequence MR imaging techniques as an alternative to CT in select patients with traumatic brain injury. ${ }^{23}$ However, this study was limited to 30 patients, often with worse presenting levels of injury, in whom the authors admit that an MR imaging may have been performed to allow prognostication or to find injuries not seen on CT that could explain the severity of neurologic injury.
However, similar to our findings, MR imaging seemed to detect intracranial injuries with similar accuracy compared with CT and had a higher accuracy in detecting diffuse axonal injuries.

No consensus exists on the clinical relevance of focal posttraumatic findings on neuroimaging studies in minor head trauma; most studies have demonstrated a correlation between intracranial hemorrhage on admission head CT with acute and long-term neuropsychiatric deficits. ${ }^{31-33}$ The disadvantages of CT, including degradation of image quality due to beam-hardening effects and displacement of the CT signal near metal objects, bone, calcifications, and high concentrations of contrast, limit the accurate assessment of brain injury. Furthermore, CT examinations performed within 3 hours of trauma may not show mature intracranial damage, thus underestimating the extent of injury. ${ }^{34}$ Full-sequence MR imaging at both $1.5 \mathrm{~T}$ and $3 \mathrm{~T}$ has shown a higher sensitivity for focal, small traumatic intracranial lesions and diffuse axonal injury. More recently, these injuries have been shown to improve outcome prediction following minor head injury. ${ }^{35}$ In a similar manner, higher sensitivity to detect these injuries in rMRI studies may allow better outcome prediction.

rMRI brain imaging allows acquisition times around 2-3 minutes, which obviates sedation or anesthesia in the pediatric patient. Motion degradation is of obvious concern when obtaining full-sequence MR imaging: In rMRI sequences in our series, 24 patients had some degree of motion artifacts on their imaging studies, compared with only 7 such cases on CT. However, despite this finding, imaging quality was adequate to reach the high level of percentage agreement seen in our study. The fastest rMRI se- 
quence, the single-shot T2 fast-field echo EPI acquired in $<5$ seconds, is almost never degraded by motion artifacts.

Decreasing the peak voltage and effective milliampere-second settings, using iterative reconstruction algorithms, and limiting the imaging area can reduce the radiation dose of a single CT examination. However, avoiding ionizing radiation altogether in the pediatric population is ideal. Many institutions across the United States have an rMRI protocol in place to image patients with shunted hydrocephalus. However, this technology is likely underused. The lack of emergency access to MR imaging facilities, lack of staffing during nights and weekends, and the inability to obtain reimbursement from third-party payers are some of the common reasons given for its underuse. ${ }^{20}$ Despite these obstacles, we have demonstrated that images can be obtained in a timely manner, and no patient had a missed lesion requiring emergency intervention. This finding is similar to that in a previous study in which 64 patients with minor head injury underwent initial evaluation with rMRI and had no clinically significant missed lesion. $^{21}$ In our study, approximately $85 \%$ of head CTs were performed between $6 \mathrm{AM}$ and $10 \mathrm{PM}$, when an in-house MR imaging technician is available. Thus, on the basis of this study of validity, a significant dose reduction in ionizing radiation could be achieved.

Our study has limitations. Initial CT scans of many patients were performed at an outside facility, with variable imaging techniques. One of the outside CTs, limited by motion artifacts, had no evidence of hemorrhage; however, hemorrhage was present on the subsequent rMRI performed at our institution. The time interval between imaging modalities may have also allowed evolution and increased conspicuity of contusions and shear injuries and an increased amount of bias. However, compared with other series, the time interval between modalities was significantly shorter.

Although only a history of trauma was provided to the interpreter in our study, the reader could assume that the studies included within the criteria had a high probability of positive findings given that a follow-up rMRI examination was indeed performed. However, for researching the validity of rMRI as an imaging technique, our institutional policy was to image all patients presenting primarily to our institution with minor head injuries with both CT and rMRI regardless of CT findings, assuming the patient met the criteria for imaging. This policy was not necessarily followed for those individuals transferred from regional institutions, who were transferred because of a positive CT finding. The radiologist interpreting the rMRI was blinded to images and the final report of the corresponding initial CT. However, in actual clinical practice, the initial CT scans are used for direct comparison during interpretation of rMRI. The availability of a comparison CT may further improve the diagnostic yield of the rMRI examination in actual clinical workflow.

\section{CONCLUSIONS}

rMRI is an adequate imaging technique for the follow-up of pediatric patients with minor head trauma. The use of rMRI could significantly reduce radiation exposure in the pediatric population.

\section{REFERENCES}

1. Luerssen TG, Klauber MR, Marshall LF. Outcome from head injury related to patient's age: a longitudinal prospective study of adult and pediatric head injury. J Neurosurg 1988;68:409-16 CrossRef Medline

2. Rutland-Brown W, Langlois JA, Thomas KE, et al. Incidence of traumatic brain injury in the United States, 2003. J Head Trauma Rehabil 2006;21:544-48 CrossRef Medline

3. Colvin JD, Thurm C, Pate BM, et al. Diagnosis and acute management of patients with concussion at children's hospitals. Arch Dis Child 2013;98:934-38 CrossRef Medline

4. Morrison J, Mâsse B, Ouellet P, et al. Four-film X-ray series is more sensitive than 2-film for diagnosis of skull fractures in children. Pediatr Emerg Care 2013;29:1189-93 CrossRef Medline

5. Af Geijerstam JL, Britton M, Marke LA. Mild head injury: observation or computed tomography? Economic aspects by literature review and decision analysis. Emerg Med J 2004;21:54-58 CrossRef Medline

6. Stein SC, Burnett MG, Glick HA. Indications for CT scanning in mild traumatic brain injury: a cost-effectiveness study. J Trauma 2006;61:558-66 CrossRef Medline

7. Sarsfield MJ, Morley EJ, Callahan JM, et al. Evaluation of emergency medicine discharge instructions in pediatric head injury. Pediatr Emerg Care 2013;29:884-87 CrossRef Medline

8. Schnadower D, Vazquez H, Lee J, et al. Controversies in the evaluation and management of minor blunt head trauma in children. Curr Opin Pediatr 2007;19:258-64 CrossRef Medline

9. Schutzman SA, Greenes DS. Pediatric minor head trauma. Ann Emerg Med 2001;37:65-74 CrossRef Medline

10. Toyama Y, Kobayashi T, Nishiyama Y, et al. CT for acute stage of closed head injury. Radiat Med 2005;23:309-16 Medline

11. Blackwell CD, Gorelick M, Holmes JF, et al. Pediatric head trauma: changes in use of computed tomography in emergency departments in the United States over time. Ann Emerg Med 2007;49: 320-24 CrossRef Medline

12. Klig JE. Issues of computerized tomography scans in children and implications for emergency care. Curr Opin Pediatr 2006;18:231-33 CrossRef Medline

13. Brenner D, Elliston C, Hall E, et al. Estimated risks of radiationinduced fatal cancer from pediatric CT. AJR Am J Roentgenol 2001; 176:289-96 CrossRef Medline

14. Malviya S, Voepel-Lewis T, Eldevik OP, et al. Sedation and general anaesthesia in children undergoing MRI and CT: adverse events and outcomes. Br J Anaesth 2000;84:743-48 CrossRef Medline

15. Coté CJ, Karl HW, Notterman DA, et al. Adverse sedation events in pediatrics: analysis of medications used for sedation. Pediatrics 2000;106:633-44 CrossRef Medline

16. Partovi S, Fram EK, Karis JP. Fast spin echo MR imaging. Neuroimaging Clin N Am 1999;9:553-76 Medline

17. Ikeda $\mathrm{K}$, Hokuto I, Mori $\mathrm{K}$, et al. Intrauterine MRI with single-shot fast-spin echo imaging showed different signal intensities in hypoplastic lungs. J Perinat Med 2000;28:151-54 Medline

18. Ashley WW Jr, McKinstry RC, Leonard JR, et al. Use of rapid-sequence magnetic resonance imaging for evaluation of hydrocephalus in children. J Neurosurg 2005;103(2 suppl):124-30 CrossRef Medline

19. O'Neill BR, Pruthi S, Bains H, et al. Rapid sequence magnetic resonance imaging in the assessment of children with hydrocephalus. World Neurosurg 2013;80:e307-312 CrossRef Medline

20. Thompson EM, Baird LC, Selden NR. Results of a North American survey of rapid-sequence MRI utilization to evaluate cerebral ventricles in children. J Neurosurg Pediatr 2014;13:636-40 CrossRef Medline

21. Missios S, Quebada PB, Forero JA, et al. Quick-brain magnetic resonance imaging for nonhydrocephalus indications. J Neurosurg Pediatr 2008;2:438-44 CrossRef Medline

22. Landis JR, Koch GG. The measurement of observer agreement for categorical data. Biometrics 1977;33:159-74 CrossRef Medline 
23. Roguski M, Morel B, Sweeney M, et al. Magnetic resonance imaging as an alternative to computed tomography in select patients with traumatic brain injury: a retrospective comparison. J Neurosurg Pediatr 2015;15:529-34 CrossRef Medline

24. Hennelly KE, Mannix R, Nigrovic LE, et al. Pediatric traumatic brain injury and radiation risks: a clinical decision analysis. $J$ Pediatr 2013;162:392-97 CrossRef Medline

25. Lee $\mathrm{H}$, Wintermark $\mathrm{M}$, Gean $\mathrm{AD}$, et al. Focal lesions in acute mild traumatic brain injury and neurocognitive outcome: CT versus 3T MRI. J Neurotrauma 2008;25:1049-56 CrossRef Medline

26. Gentry LR, Godersky JC, Thompson B, et al. Prospective comparative study of intermediate-field MR and CT in the evaluation of closed head trauma. AJR Am J Roentgenol 1988;150:673-82 CrossRef Medline

27. Jenkins A, Teasdale G, Hadley MD, et al. Brain lesions detected by magnetic resonance imaging in mild and severe head injuries. Lancet 1986;2:445-46 Medline

28. Mittl RL, Grossman RI, Hiehle JF, et al. Prevalence of MR evidence of diffuse axonal injury in patients with mild head injury and normal head CT findings. AJNR Am J Neuroradiol 1994;15:1583-89 Medline

29. Orrison WW, Gentry LR, Stimac GK, et al. Blinded comparison of cranial CT and MR in closed head injury evaluation. AJNR Am J Neuroradiol 1994;15:351-56 Medline
30. Yuh EL, Mukherjee P, Lingsma HF, et al; TRACK-TBI Investigators Magnetic resonance imaging improves 3-month outcome prediction in mild traumatic brain injury. Ann Neurol 2013;73:224-35 CrossRef Medline

31. Smith-Bindman R, Lipson J, Marcus R, et al. Radiation dose associated with common computed tomography examinations and the associated lifetime attributable risk of cancer. Arch Intern Med 2009; 169:2078-86 CrossRef Medline

32. Linton OW, Mettler FA Jr; National Council on Radiation Protection and Measurements. National conference on dose reduction in CT, with an emphasis on pediatric patients. AJR Am J Roentgenol 2003; 181:321-29 CrossRef Medline

33. White KS. Invited article: helical/spiral CT scanning-a pediatric radiology perspective. Pediatr Radiol 1996;26:5-14 CrossRef Medline

34. Kashluba S, Hanks RA, Casey JE, et al. Neuropsychologic and functional outcome after complicated mild traumatic brain injury. Arch Phys Med Rehabil 2008;89:904-11 CrossRef Medline

35. Sadowski-Cron C, Schneider J, Senn P, et al. Patients with mild traumatic brain injury: immediate and long-term outcome compared to intra-cranial injuries on CT scan. Brain Inj 2006;20:1131-37 CrossRef Medline 